\title{
Comparative analysis of Etest and broth microdilution method (AFST-EUCAST) for trends in antifungal drug susceptibility testing of Brazilian Cryptococcus neoformans isolates
}

\begin{abstract}
Correspondence
Claudete R. Paula

crpmicol@uol.com.br
\end{abstract}

Received 18 June 2006

Accepted 15 August 2006

\author{
Amanda L. T. Dias, ${ }^{1,2}$ Flavia E. Matsumoto, ${ }^{1}$ Marcia S. C. Melhem, ${ }^{3}$ \\ Eriques G. da Silva, ${ }^{1}$ Marcos E. Auler, ${ }^{1}$ Antonio M. de Siqueira ${ }^{2}$ \\ and Claudete R. Paula ${ }^{1}$
1Departamento de Microbiologia, Laboratório de Micologia, Instituto de Ciências Biomédicas II, Universidade de São Paulo (USP), São Paulo-SP, Brazil
${ }^{2}$ Departamento de Ciências Biológicas, Laboratório de Microbiologia, Universidade Federal de Alfenas-MG, Alfenas-MG, Brazil
${ }^{3}$ Instituto Adolfo Lutz, São Paulo-SP, Brazil

\begin{abstract}
A prospective study was performed to evaluate the correlation between the proposed standard of the Antifungal Susceptibility Testing Subcommittee of the European Committee on Antibiotic Susceptibility Testing (AFST-EUCAST) (document 7.1) and the commercial system Etest for determining the MICs of flucytosine, amphotericin B, fluconazole, itraconazole and voriconazole for a collection of 100 clinical and environmental isolates of Cryptococcus neoformans. The agreements among Etest MICs within $\pm 2 \log _{2}$ dilutions of AFST-EUCAST standard MICs were greater for flucytosine, fluconazole and voriconazole (76, 78 and $88 \%$, respectively) than for amphotericin B (5\%), the lowest agreement, and itraconazole (67\%). Overall, the correlation coefficients were statistically significant $(P<0.05)$, and it is suggested that the Etest and AFST-EUCAST method are reliable alternatives and present good correlation for all drugs evaluated except amphotericin B. However, the observed differences related to MICs for susceptible, susceptible dose dependent and resistant strains between the methods suggest that it will be necessary to carry out further studies, including assessment of interlaboratory agreement and correlation of MICs by different methods with in vivo response.
\end{abstract}

\section{INTRODUCTION}

Cryptococcus neoformans is ubiquitous in the environment, and usually it is found associated with avian guano and vegetative debris (Randhawa et al., 2003). The yeast is an exogenous and opportunistic pathogen capable of causing life-threatening infections, especially in people with immunodeficiencies, such as patients with AIDS, transplanted organs or some haematological malignancies (Casadevall \& Perfect, 1998). The pharmacological management of cryptococcal infections usually consists of primary therapy with amphotericin B, with or without flucytosine, almost always followed by a maintenance therapy, or a life-long suppressive therapy, with some azoles, principally, fluconazole (Saag et al., 2000). High rates of fungal persistence and frequent disease relapse have sparked an increasing

Abbreviations: AFST-EUCAST, Antifungal Susceptibility Testing Subcommittee of the European Committee on Antibiotic Susceptibility Testing; CLSI, Clinical and Laboratory Standards Institute; R, resistant; S, susceptible; S-DD, susceptible dose-dependent. concern among clinicians considering the potential for antifungal resistance emergence among $C$. neoformans strains (Berg et al., 1998; Brandt et al., 2001). For these reasons it is necessary to develop routine methods for determining the susceptibility of $C$. neoformans strains isolated from different sites to antifungal agents in order to evaluate the efficacy of treatments and the evolution of the disease. The main effort to develop a routine method for testing susceptibility of yeasts to antifungal agents has employed different species of Candida. These methods for determining the in vitro susceptibility of Candida spp., however, cannot be extrapolated to C. neoformans, owing to the fastidious nature of this micro-organism and its slow growth (Ghannoun et al., 1992). The development of standardized antifungal susceptibility testing methods for the latter fungi has been the subject of numerous studies during the last decade (Pfaller et al., 2001; Rex et al., 2001). The Antifungal Susceptibility Testing Subcommittee of the European Committee on Antibiotic Susceptibility Testing (AFST-EUCAST) has proposed a standard for the 
determination of fermentative yeast MICs and C. neoformans MICs by a broth dilution technique (Antifungal Susceptibility Testing Subcommittee of the European Committee on Antibiotic Susceptibility Testing, 2002). The proposed standard of the AFST-EUCAST is based on the reference Clinical and Laboratory Standards Institute (CLSI) (formerly the National Committee for Clinical Laboratory Standards) M27-A2 procedures (Clinical and Laboratory Standards Institute, 2002) but it incorporates some modifications in order to get an objective and automated antifungal susceptibility testing and to shorten the incubation time for MIC determination. The modifications include the use of RPMI 1640 supplemented with $2 \%$ glucose as assay medium, an inoculum size of $0.5 \times 10^{5}$ $2 \cdot 5 \times 10^{5}$ c.f.u. $\mathrm{ml}^{-1}$, flat-bottom trays, spectrophotometric reading, $50 \%$ inhibition as the end point for azole agents and flucytosine, and $90 \%$ inhibition as the end point for amphotericin B. In recent reports it has been pointed out that susceptibility results obtained by using the proposed standard of EUCAST show high percentages of agreement with those obtained by the CLSI reference procedure (Clinical and Laboratory Standards Institute, 2002). However, the agreement among susceptibility testing methods for $C$. neoformans has not been evaluated for many antifungal agents. Several reports indicate that the Etest is a viable alternative to the reference methods for susceptibility testing of yeasts. Procedures based on Etest strips are straightforward, economical to perform, and offer a practical method for a clinical laboratory (Chryssanthou et al., 1997; Warnock et al., 1998). The purpose of this study was to compare the results obtained by the proposed AFSTEUCAST procedure with those obtained with the Etest system (AB BIODISK) for determination of the susceptibilities of 100 Cryptococcus species isolates to 5 antifungal agents.

\section{METHODS}

Isolates. One hundred isolates of $C$. neoformans (all being from serotype A) were selected for testing. The choice was made according to previous results (data not shown) of tests to study the presence of factors related to the virulence of the yeast, like phospholipase, proteinase, phenoloxidase and superoxide dismutase. Amongst the obtained results, samples with high, intermediate and low enzyme production were selected for the research of the antifungal susceptibility profile of Brazilian isolates of C. neoformans. These isolates (50 clinical and 50 environmental ones) were from geographically diverse regions in Brazil. The clinical ones had all been isolated from either cerebrospinal fluid or blood samples from infected patients and the environmental isolates were obtained from avian guanos and hollow trees. Prior to testing, each isolate was subcultured at least twice on Sabouraud dextrose agar plates (Difco) to ensure purity and optimal growth. C. neoformans ATCC 90112 was included as a control strain (Aller et al., 2000).

Antifungal susceptibility testing. We employed as the gold standard methodology the broth microdilution procedure described in document 7.1 by Antifungal Susceptibility Testing Subcommittee of the European Committee on Antibiotic Susceptibility Testing (2002) that presents a high correlation with the document 27-A2 proposed by the Clinical and Laboratory Standards Institute (2002).
AFST-EUCAST procedures. The AFST-EUCAST broth microdilution procedure was performed according to the published method (Antifungal Susceptibility Testing Subcommittee of the European Committee on Antibiotic Susceptibility Testing, 2002). A yeast inoculum of $0 \cdot 5 \times 10^{5}$ to $2 \cdot 5 \times 10^{5}$ c.f.u. $\mathrm{ml}^{-1}$ was prepared in sterile water and validated by colony counts. The assay medium was a double-strength solution of RPMI 1640 with L-glutamine and without bicarbonate, buffered to $\mathrm{pH} 7 \cdot 0$ with $0 \cdot 165 \mathrm{M}$ MOPS and supplemented with $18 \mathrm{~g}$ glucose $1^{-1}$. Twofold serial dilutions of antifungal drugs were prepared in microdilution trays that contained $100 \mu \mathrm{l}$ inoculum with between $0.5 \times 10^{5}$ and $2 \cdot 5 \times 10^{5}$ c.f.u. $\mathrm{ml}^{-1}$. All drugs were obtained as reagent grade powders: flucytosine and amphotericin B from Sigma, fluconazole from Pfizer, itraconazole from Janssen-Cilag and voriconazole from Pfizer. The final concentrations were $0.03-16 \mathrm{mg} \mathrm{l}^{-1}$ for flucytosine and amphotericin $\mathrm{B}$, $0 \cdot 12-64 \mathrm{mg} \mathrm{l}^{-1}$ for fluconazole, $0 \cdot 015-8 \mathrm{mg} \mathrm{l}^{-1}$ for itraconazole and voriconazole. The microdilution plates were incubated at $35^{\circ} \mathrm{C}$ in a non- $\mathrm{CO}_{2}$ incubator, and MICs were determined with a spectrophotometer after $48 \mathrm{~h}$; microdilution plates were reincubated if the $\mathrm{OD}_{500}$ was $\leqslant 0.5$ (indicative of poor growth) and read after the next day of incubation. For flucytosine and the azoles (fluconazole, itraconazole and voriconazole), the MIC end point was defined as the lowest drug concentration resulting in a reduction of growth of $50 \%$ or more compared with growth of the control. For amphotericin $\mathrm{B}$, the end point was the lowest concentration that resulted in a reduction in growth of $90 \%$ or more compared with growth of the control (Cuenca-Estrella et al., 2003; Espinel-Ingroff et al., 2005). The MICs at which $50 \%$ (geometric mean $\mathrm{MIC}$ ) and $90 \%\left(\mathrm{MIC}_{90}\right)$ of each of the 100 isolates tested suffered inhibition were determined for each drug.

Etest procedures. Etest strips (AB BIODISK) containing flucytosine, amphotericin $\mathrm{B}$, fluconazole, itraconazole and voriconazole were purchased from Probac. The concentration gradient for flucytosine, amphotericin $\mathrm{B}$, itraconazole and voriconazole ranged from 0.002 to $32 \mu \mathrm{g} \mathrm{ml}^{-1}$ and for fluconazole ranged from 0.016 to $256 \mu \mathrm{g} \mathrm{ml}^{-1}$. The strips were stored at $-20{ }^{\circ} \mathrm{C}$ until needed. The agar formulation used for the Etest was RPMI 1640 (American Biorganics) supplemented with $1.5 \%$ agar and $2 \%$ glucose, and buffered with MOPS (Lozano-Chiu et al., 1998; Pfaller et al., 2000). Plates of $90 \mathrm{~mm}$ diameter containing RPMI 1640 and $2 \%$ glucose agar to a depth of $4.0 \mathrm{~mm}$ were used. The agar surface was inoculated by using a non-toxic swab dipped in a cell suspension adjusted spectrophotometrically to the turbidity of a $1.0 \mathrm{McF}$ arland standard. After the excess moisture was absorbed into the agar and the surface was completely dry, Etest strips were applied to each plate. The plates were incubated at $35^{\circ} \mathrm{C}$ and read at 48 and $72 \mathrm{~h}$. The azole MICs were read at the lowest concentration at which the border of the elliptical inhibition zone intercepted the scale on the strip. Any growth, such as microcolonies, throughout a discernible inhibition ellipse was ignored (Pfaller et al., 2000). The flucytosine and amphotericin B MICs were read at the point at which the zone of almost complete inhibition intersected the strip. Sometimes, the growth of microcolonies up to the strip could be observed, principally in the case of azoles. So, the interpretation of the results must be made according to the criteria documented in the Etest technical guide (www.abbiodisk.com) by selecting the MIC value as the point of approximately $80 \%$ of growth inhibition to azoles and the point of almost complete inhibition (95\%) of growth for flucytosine and amphotericin B.

Quality control. All susceptibility tests were repeated twice. The evaluation of antifungal susceptibility by both methodologies were realized simultaneously to avoid sample maintenance for different periods between the realization of one test and another. The potency and viability of stock solutions and Etest strips were controlled. 
Periodic evaluations were realized with standard C. neoformans samples, like ATCC 90112, whose antifungal susceptibility profile has been previously established.

Interpretation of results. The interpretative criteria used for susceptibility to flucytosine, fluconazole and itraconazole were those published by the CLSI (M27-A2) (Clinical and Laboratory Standards Institute, 2002): for flucytosine, susceptible (S) $\leqslant 4 \mu \mathrm{g} \mathrm{ml}^{-1}$; intermediate (I) $8-16 \mu \mathrm{g} \mathrm{ml}^{-1}$; resistant (R) $\geqslant 32 \mu \mathrm{g} \mathrm{ml}^{-1}$; for fluconazole, $\mathrm{S} \leqslant 8 \mu \mathrm{g} \mathrm{ml}^{-1}$; susceptible dose dependent (S-DD) $16-32 \mu \mathrm{g} \mathrm{ml}^{-1} ; \mathrm{R} \geqslant 64 \mu \mathrm{g} \mathrm{ml}^{-1}$; for itraconazole, $\mathrm{S} \leqslant 0 \cdot 125 \mu \mathrm{g} \mathrm{ml}^{-1} ;$ S-DD $0 \cdot 25-0.5 \mu \mathrm{g} \mathrm{ml}^{-1} ; \mathrm{R} \geqslant 1 \mu \mathrm{g} \mathrm{ml}^{-1}$. Interpretative criteria have not yet been approved for amphotericin B. As suggested by Nguyen \& Yu (1998) and Lozano-Chiu et al. (1998) isolates for which amphotericin B MICs were $\geqslant 2 \mu \mathrm{g} \mathrm{ml}^{-1}$ were considered resistant $\left(\leqslant 1 \mu \mathrm{g} \mathrm{ml}^{-1}\right.$, susceptible). For purposes of comparison, and because preliminary pharmacokinetic data indicated that levels of voriconazole achievable in serum may range from 2 to $6 \mu \mathrm{g} \mathrm{ml}^{-1}$ (Andes, 2003; Andes et al., 2003; Groll \& Kolve, 2004; Petraitiene et al., 2001), we have used a breakpoint for susceptibility of $\leqslant 1 \mu \mathrm{g} \mathrm{ml}^{-1}$ and for resistance $\geqslant 4 \mu \mathrm{g} \mathrm{ml}^{-1}$ (Cuenca-Estrella et al., 2003; Clinical and Laboratory Standards Institute, 2005).

Data analysis. Statistical analysis was done through the evaluation of the Pearson correlation coefficient by Analyse-it (version 1.73, Analyse-it Software) correlating Etest MICs with AFST-EUCAST MICs. The Pearson correlation, also known as the linear or productmoment correlation, is a parametric test to measure how linearly related the variables are. The degree of association is expressed by the correlation coefficient $(r)$. The tests are highly correlated when $r>0 \cdot 9$. The Etest and AFST-EUCAST MICs curves can be compared if the area under the curve is inside the range $0 \cdot 7-0 \cdot 975$. So, if $r$ is inside the values cited and $P<0 \cdot 05$, the two groups are significantly correlated. Etest and AFST-EUCAST MIC ranges and corresponding geometric mean values were analysed for each species-drug combination. Etest and AFST-EUCAST MIC ${ }_{90}$ were also determined.

Both on-scale and off-scale results were included in the analysis because the Etest strips contain a continuous gradient of each drug tested instead of the $\log _{2}$ drug dilution scheme of the broth microdilution methods. So the Etest MICs were elevated to the next drug concentration that matched the microdilution scheme to facilitate comparison of the results. The percentage of agreement between the Etest and the AFST-EUCAST methodology was defined as the proportion of Etest results that fell within $\pm 2 \log _{2}$ dilutions of the EUCAST MIC results. So, values were considered in agreement when the discrepancies between the methods were no more than $2 \log _{2}$ dilutions. In addition, the Etest results were examined to determine whether this method tended to produce higher or lower MICs than the AFST-EUCAST method.

The interpretative classifications of the isolates yielded by each test method were compared and discrepancies were defined as follows: (i) minor, isolate classified as S or R by one of the tests and as S-DD or I by the other method; (ii) major, isolate classified as S by AFST-EUCAST method and R by Etest; and (iii) very major, isolate classified as R by AFST-EUCAST method and S by Etest.

\section{RESULTS AND DISCUSSION}

Table 1 depicts the susceptibilities of 100 C. neoformans isolates to 5 antifungal agents through the determination of AFST-EUCAST method and Etest MIC ranges and corresponding geometric mean MICs for each speciesdrug combination. The comparison of MICs obtained by both methodologies resulted in a considerable correlation index varying from $0 \cdot 92$, as in the case of flucytosine, to $0 \cdot 7$, as seen for voriconazole. The overall levels of agreement among the results (Etest MICs within $\pm 2 \log _{2}$ dilutions of EUCAST MICs) were greater for voriconazole $(88 \%)$, fluconazole $(78 \%)$, flucytosine $(76 \%)$, itraconazole $(67 \%)$ than for amphotericin B (5\%), which has the lowest level of agreement, as already seen by other authors (Aller et al., 2000) (Table 2). The in vitro interpretative antifungal susceptibility classification of the C. neoformans isolates as $\mathrm{S}, \mathrm{S}-\mathrm{DD}$ and $\mathrm{R}$ is summarized in Table 3 (Clinical and Laboratory Standards Institute, 2002). According to both methodologies, MICs for flucytosine, fluconazole, itraconazole and voriconazole tended to be clustered within the upper limits of the S category and in the S-DD and/or I

Table 1. In vitro susceptibilities of 100 Cryptococcus spp. isolates to five antifungal agents as determined by AFST-EUCAST and Etest methods, and Pearson correlation among MIC values obtained by each method

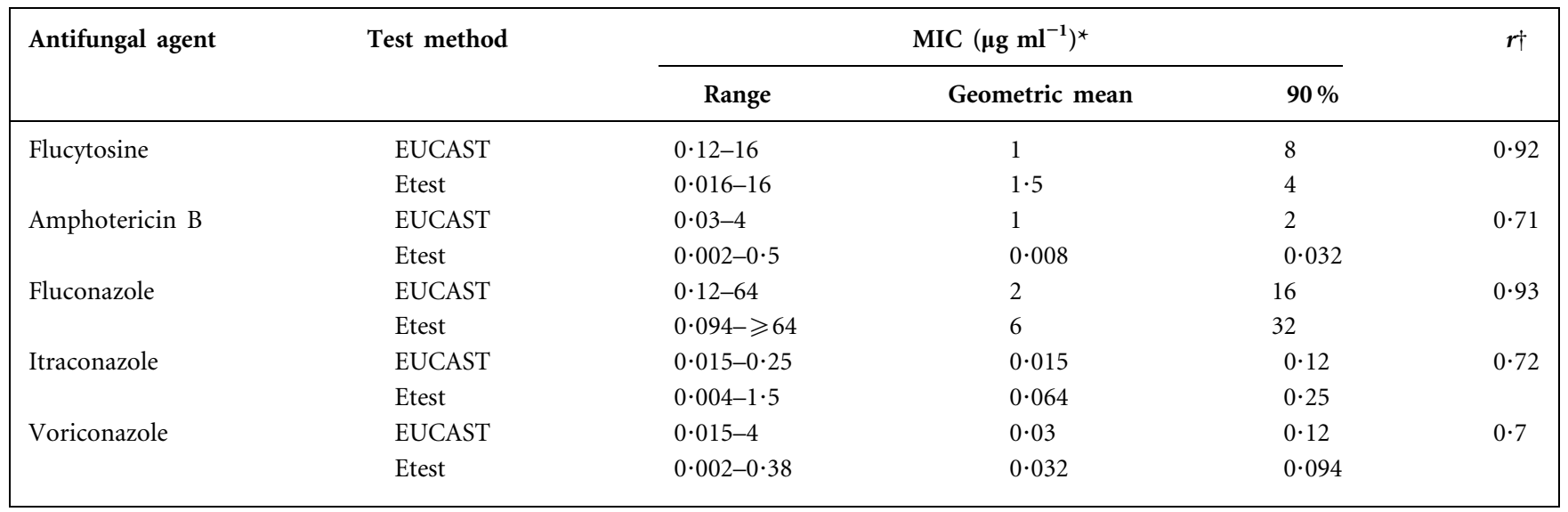

$\star 50$ and $90 \%$ MICs that inhibited 50 and $90 \%$ of the isolates tested, respectively.

$\dagger$ Pearson correlation coefficient (if the area under the curve corresponds to $0 \cdot 7<r<0 \cdot 975$ the two curves can be compared for $P<0 \cdot 001$ ). 
Table 2. Distribution of differences in MICs for 100 C. neoformans isolates and percentage agreement within 3 dilutions for the AFST-EUCAST and Etest methods

\begin{tabular}{|c|c|c|c|c|c|c|c|c|}
\hline \multirow[t]{2}{*}{ Antifungal agent } & \multicolumn{7}{|c|}{ No. of isolates with Etest MICs different from the AFST-EUCAST method MICs } & \multirow{2}{*}{$\begin{array}{c}\text { Percentage MIC agreement } \\
\quad \pm 2 \log _{2} \text { dilution }\end{array}$} \\
\hline & $>+2$ & +2 & +1 & 0 & -1 & -2 & -2 & \\
\hline Flucytosine & 10 & 11 & 13 & 29 & 8 & 15 & 14 & 76 \\
\hline Amphotericin B & - & - & - & 1 & 2 & 2 & 95 & 5 \\
\hline Fluconazole & 22 & 22 & 12 & 23 & 7 & 13 & 1 & 78 \\
\hline Itraconazole & 29 & 17 & 17 & 20 & 13 & 2 & 2 & 67 \\
\hline Voriconazole & 3 & 9 & 14 & 35 & 20 & 12 & 7 & 88 \\
\hline
\end{tabular}

${ }^{*}$ Percentage of agreement between the results is defined as the proportion of Etest MIC results that were within $\pm 2 \log _{2}$ dilutions of the broth microdilution MIC results.

category. Voriconazole and fluconazole showed the greatest category agreement. No one putative isolate resistant to flucytosine was observed while the same number of resistant isolates was observed for fluconazole by both methodologies. None of the classifications for flucytosine and itraconazole yielded by the alternative methodology Etest represented 'very major' discrepancies with respect to the AFST-EUCAST proposed one. The greatest percentage of 'very major' discrepancies was observed for amphotericin B, where $30 \%$ of the isolates fell into this category. (Hitchcock et al., 1995) (Table 3).

Since yeast infections are increasing and more resistant strains are observed (Revankar et al., 1996; Schwarz et al., 2006), a comparison of the currently available routine methods for susceptibility testing among themselves and/or with a gold standard method has been required (Vandenbossche et al., 2002).

This is the first study in which the AFST-EUCAST proposed method has been compared with the Etest technique for flucytosine, amphotericin B, fluconazole, itraconazole and voriconazole susceptibility testing.

Warnock et al. (1998) found that the MICs by Etest for amphotericin B were lower than the results obtained with the reference microdilution method, whereas the Etest MICs results for flucytosine, fluconazole and itraconazole were higher than those obtained with the microdilution method. Other authors (Brass et al., 1979; Odds, 1980) have reported that absolute azole MICs generated by agar-based techniques tend to be lower than those produced by broth assays. In agreement with other authors (Colombo et al.,

Table 3. Interpretative antifungal susceptibility classification of 100 Cryptococcus isolates determined by AFST-EUCAST and Etest methods

\begin{tabular}{|c|c|c|c|c|c|c|c|c|}
\hline \multirow[t]{2}{*}{ Antifungal agent } & \multirow[t]{2}{*}{ Method } & \multicolumn{3}{|c|}{ Percentage of isolates by category ${ }^{\star}$} & \multicolumn{3}{|c|}{ Percentage of discrepancy results $\dagger$} & \multirow[t]{2}{*}{$\%$ Category agreement $\ddagger$} \\
\hline & & $\mathbf{S}$ & S-DD and/or I & $\mathbf{R}$ & Minor & Major & Very major & \\
\hline \multirow[t]{2}{*}{ Flucytosine } & EUCAST & 86 & 14 & 0 & 10 & 0 & 0 & 90 \\
\hline & Etest & 93 & 7 & 0 & & & & \\
\hline \multirow[t]{2}{*}{ Amphotericin B } & EUCAST & 70 & 0 & 30 & 0 & 0 & 30 & 70 \\
\hline & Etest & 100 & 0 & 0 & & & & \\
\hline \multirow[t]{2}{*}{ Fluconazole } & EUCAST & 89 & 8 & 3 & 23 & 3 & 0 & 74 \\
\hline & Etest & 74 & 23 & 3 & & & & \\
\hline \multirow[t]{2}{*}{ Itraconazole } & EUCAST & 95 & 5 & 0 & 11 & 0 & 0 & 89 \\
\hline & Etest & 91 & 8 & 1 & & & & \\
\hline \multirow[t]{2}{*}{ Voriconazole } & EUCAST & 99 & 0 & 1 & 0 & 0 & 1 & 99 \\
\hline & Etest & 100 & 0 & 0 & & & & \\
\hline
\end{tabular}

${ }^{*}$ Percentage of isolates classified in the given categories, see Methods for definitions.

$\dagger$ Percentage of the results representing minor, major or very major discrepancies with respect to those of the AFST-EUCAST method, see Methods for definitions.

¥Agreement rates reflect the percentage of isolates classified in the same category by the proposed AFST-EUCAST method and the alternative Etest method. 
1995; Espinell-Ingroff et al., 1996) we observed the growth of microcolonies around or inside the inhibition zone when we tested flucytosine and fluconazole. The correlation of the Etest with the AFST-EUCAST method was acceptable, and to the best of our knowledge such a correlation for susceptibility testing for $C$. neoformans to these drugs has not been published yet. The correlations between the methods were acceptable since the $r$ values were inside the range $0 \cdot 7-0 \cdot 975$. The greatest values of $r$ for flucytosine and fluconazole indicated that the MICs for these drugs varied in a similar range by both methodologies despite the results obtained to the other drugs that presented lower $r$ values. The MIC ranges for amphotericin $\mathrm{B}$, itraconazole and voriconazole were not similar by the two methodologies (Table 1). Colombo et al. (1995) obtained a similar range by CLSI and Etest methods for fluconazole and itraconazole. The MICs obtained by Aller et al. (2000) with Etest had larger ranges than ours except for amphotericin B, which had narrower ranges. Etest geometric mean MIC and $\mathrm{MIC}_{90}$ values were greater for flucytosine and amphotericin B and lower for fluconazole. The Etest geometric mean MIC for itraconazole was greater and the $\mathrm{MIC}_{90}$ was equal to the CLSI MIC, as was also observed by Abdel Salam (2005), who obtained greater values for amphotericin B and lower values of geometric mean MIC for flucytosine, fluconazole, itraconazole. Overall, the agreement between methodologies was considered significant. The highest agreement $\left( \pm 2 \log _{2}\right)$ between methods was seen for voriconazole $(88 \%)$ as was also observed by Chryssanthou et al. (1997) for Candida spp. (90\%). For amphotericin B we observed a low agreement (5\%) between the MICs obtained by the two methodologies. Aller et al. (2000) found a correlation of $13.5 \%$ for amphotericin B MIC in comparison of Etest and CLSI methods. We found 30 resistant isolates (30\%) to this drug by the AFST-EUCAST method and none by Etest. Our results contrasted with the ones obtained by Perkins et al. (2005) where the rates of antifungal resistance for amphotericin B were $5 \cdot 3 \%$. Warnock et al. (1998) tested 18 isolates of Candida spp. and 2 C. neoformans isolates against amphotericin B, flucytosine, fluconazole and itraconazole by the Etest method in a multi-centre evaluation. These authors suggested that the Etest method is suitable for routine use with Candida spp. in evaluations for amphotericin B and flucytosine, but it is less reliable for the azoles. However, the two $C$. neoformans isolates generated a large number of discrepant results, which is in agreement with results obtained by Aller et al. (2000). In that work the greatest discrepancies were with amphotericin B $(8 \cdot 1 \%$ agreement on antibiotic medium 3 and $13 \cdot 5 \%$ agreement on RPMI 1640). These results disagree with those obtained by Lozano-Chiu et al. (1998) who evaluated the CLSI M27-A microdilution and the Etest agar diffusion methods for the detection of resistance to amphotericin B among C. neoformans isolates. For the putatively susceptible isolates they obtained results by Etest on RPMI 1640 agar and antibiotic medium 3 agar similar to those obtained for antibiotic medium 3 by microdilution, whereas the MICs for the resistant isolates were noticeably increased. Aller et al. (2000) found all strains putatively susceptible to amphotericin B and did not find a correlation between the values obtained by the two methods like in our study. The activity of voriconazole was the best one against C. neoformans. Voriconazole, a derivative of fluconazole, has previously been demonstrated to have a greater activity compared with itraconazole, and a broader spectrum of activity and higher potency than fluconazole against Candida spp., C. neoformans, Aspergillus spp. and other fungi (Hoban et al., 1999). Voriconazole has recently been used for the treatment of several difficult-to-treat fungal infections, including refractory cryptococcal meningitis (Johnson \& Kauffman, 2003; Perfect et al., 2003). In comparison to flucytosine, amphotericin $\mathrm{B}$, fluconazole and itraconazole, voriconazole has the lowest MIC for $C$. neoformans (Espinel-Ingroff, 1998; McGinnis et al., 1997; Nguyen \& Yu, 1998; Pfaller et al., 1999; Yildiran et al., 2002). Limited animal studies have shown that voriconazole is effective in reducing $C$. neoformans c.f.u. levels in lung and brain tissues in pulmonary and intracranial models, respectively (Hitchcock et al., 1995; Van Duin et al., 2004). The recent emergence of $C$. neoformans isolates with decreased susceptibilities to fluconazole and amphotericin $\mathrm{B}$ is of great concern because these agents are recommended as the initial drugs of choice for the treatment of cryptococcosis. So, routine susceptibility testing of $C$. neoformans isolates for guidance during initial antifungal therapy is warranted (Aller et al., 2000, Saag et al., 2000). In conclusion, we suggest the Etest and AFST-EUCAST methods are reliable alternatives and presented good correlation to all drugs we evaluated except to amphotericin B. For fluconazole the detection of resistance by both methods correlated highly. On the contrary, for the detection of 31 resistant isolates by the AFST-EUCAST method (30 for amphotericin B and 1 for voriconazole) against only 1 for itraconazole by Etest there was a diminished correlation between the methods. Some isolates that tested resistant for amphotericin B and voriconazole by the AFST-EUCAST method appeared to be susceptible when tested by the Etest method. Generally this happens with such agar-based methods because of the trailing growth associated with broth methods like the AFSTEUCAST one. On the basis of our data we suggest that the results for isolates that appear to be resistant by any method should be carefully reviewed and such isolates may merit repeat testing and/or testing by other methods. Although more work needs to be done with less susceptible isolates, the aggregate data suggest that agar-based methods appear to produce a more consistent in vitro-in vivo correlation than the broth-based one by eliminating the trailing growth from the equation. However, the observed differences in MICs for S, S-DD and R strains between methods suggest that it will be necessary to carry out further studies, including assessment of interlaboratory agreement and correlation of MICs by different methods with in vivo response.

\section{ACKNOWLEDGEMENTS}

This work was supported by FAPESP (02/07296-8 and 04/05407-2). 


\section{REFERENCES}

Abdel Salam, H. A. (2005). In vitro susceptibility of Cryptococcus neoformans clinical isolates from Egypt to seven antifungal drugs. Mycoses 48, 327-32.

Aller, A. I., Martin-Mazuelos, E., Lozano, F., Gomez-Mateos, J., Steele-Moore, L., Holloway, W. J., Gutiérrez, M. J., Recio, F. J. \& Espinel-Ingroff, A. (2000). Correlation of fluconazole MICs with clinical outcome in cryptococcal infection. Antimicrob Agents Chemother 44, 1544-1548.

Andes, D. (2003). In vivo pharmacodynamics of antifungal drugs in treatment of candidiasis. Antimicrob Agents Chemother 47, 1179-1186.

Andes, D., Marcillo, K., Stamstad, T. \& Conklin, R. (2003). In vivo pharmacokinetics and pharmacodynamics of a new triazole, voriconazole, in a murine candidiasis model. Antimicrob Agents Chemother 47, 3165-3169.

Antifungal Susceptibility Testing Subcommittee of the European Committee on Antibiotic Susceptibility Testing (2002). Method for Determination of Minimal Inhibitory Concentration (MIC) by Broth Dilution of Fermentative Yeast, discussion document E.Dis7.1. Taufkirchen: European Society of Clinical Microbiology and Infectious Diseases.

Berg, J., Clancy, C. J. \& Nguyen, M. H. (1998). The hidden danger of primary fluconazole prophylaxis for patients with AIDS. Clin Infect Dis 26, 186-187.

Brandt, M. E., Pfaller, M. A., Hajjeh, R. A., Hamill, R. J., Pappas, P. G., Reingold, A. L., Rimland, D. \& Warnock, D. W. for the Cryptococcal Disease Active Surveillance Group (2001). Trends in antifungal drug susceptibility of Cryptococcus neoformans isolates in the United States: 1992 to 1994 and 1996 to 1998. Antimicrob Agents Chemother 45, 3065-3069.

Brass, C., Shainhouse, J. Z. \& Stevens, D. A. (1979). Variability of agar dilution-replicator method of yeast susceptibility testing. Antimicrob Agents Chemother 15, 763-768.

Casadevall, A. \& Perfect, J. R. (1998). Cryptococcus neoformans, 1st edn. Washington, DC: American Society for Microbiology.

Chryssanthou, E., Gronfors, C. \& Khanna, N. (1997). Comparison of broth macrodilution, broth microdilution and E-test susceptibility tests of Cryptococcus neoformans for fluconazole. Mycoses 40, 423-427.

Clinical and Laboratory Standards Institute (2002). Reference Method for Broth Dilution Antifungal Susceptibility Testing of Yeasts, Approved Standard, 2nd edn, M27-A2. Wayne, PA: Clinical and Laboratory Standards Institute.

Clinical and Laboratory Standards Institute (2005). Quality Control Minimal Inhibitory Concentration (MIC) Limits for Broth Microdilution and MIC Interpreative Breakpoints, M27-S2. Wayne, PA: Clinical and Laboratory Standards Institute.

Colombo, A. L., Barchiesi, F., McGough, D. A. \& Rinaldi, M. G. (1995). Comparison of Etest and National Committee for Clinical Laboratory Standards broth macrodilution method for azole antifungal susceptibility testing. J Clin Microbiol 33, 535-540.

Cuenca-Estrella, M., Moore, C. B., Barchiesi, F. \& 12 other authors (2003). Multicenter evaluation of the reproducibility of the proposed antifungal susceptibility method for fermentative yeasts of the Antifungal Susceptibility Testing Subcommittee of the European Committee on Antimicrobial Susceptibility Testing (AFSTEUCAST). Clin Microbiol Infect 9, 467-474.

Espinel-Ingroff, A. (1998). In vitro activity of the new triazole voriconazole (UK-109,496) against opportunistic filamentous and dimorphic fungi and common and emerging yeast pathogens. J Clin Microbiol 36, 198-202.
Espinell-Ingroff, A., Pfaller, M., Erwin, M. E. \& Jones, R. N. (1996). Interlaboratory evaluation of Etest method for testing antifungal susceptibilities of pathogenic yeast to five antifungal agents by using Casitone agar and solidified RPMI 1640 medium with 2\% glucose. J Clin Microbiol 34, 848-852.

Espinel-Ingroff, A., Barchiesi, F., Cuenca-Estrella, M., Pfaller, M. A., Rinaldi, M., Rodriguez-Tudela, J. L. \& Verweij, P. E. (2005). International and multicenter comparison of EUCAST and CLSI M27-A2 broth microdilution methods for testing susceptibilities of Candida spp. to fluconazole, itraconazole, posaconazole, and voriconazole. J Clin Microbiol 43, 3884-3889.

Ghannoun, M. A., Ibrahim, A. S., Fu, Y., Shafiq, M. C., Edwards, J. E. \& Criddle, R. S. (1992). Susceptibility testing of Cryptococcus neoformans: a microdilution technique. J Clin Microbiol 30, 2881-2886.

Groll, A. H. \& Kolve, H. (2004). Antifungal agents: in vitro susceptibility testing, pharmacodynamics, and prospects for combination therapy. Eur J Clin Microbiol Infect Dis 23, 256-270.

Hitchcock, C. A., Andrews, R. J., Lewis, B. G. H. \& Troke, P. F. (1995). UK-109,496, a novel, wide-spectrum triazole derivative for the treatment of fungal infections: antifungal activity in experimental infections with Cryptococcus. In Abstracts of the 35th Interscience Conference on Antimicrobial Agents and Chemotherapy, abstract F75, p. 126. Washington, DC: American Society for Microbiology.

Hoban, D. J., Zhanel, G. G. \& Karlowsky, J. A. (1999). In vitro susceptibilities of Candida and Cryptococcus neoformans isolates from blood cultures of neutropenic patients. Antimicrob Agents Chemother 43, 1463-1464.

Johnson, L. B. \& Kauffman, C. A. (2003). Voriconazole: a new triazole antifungal agent. Clin Infect Dis 36, 630-637.

Lozano-Chiu, M., Paetznick, V. L., Ghannoum, M. A. \& Rex, J. H. (1998). Detection of resistance to amphotericin B among Cryptococcus neoformans clinical isolates: performance of three different media assessed by using E-Test and National Committee for Clinical Laboratory Standards M27-A methodologies. J Clin Microbiol 36, 2817-2822.

McGinnis, M. R., Pasarell, L., Sutton, D. A., Fothergill, A. W., Cooper, R., Jr \& Rinaldi, M. G. (1997). In vitro evaluation of voriconazole against some clinically important fungi. Antimicrob Agents Chemother 41, 1832-1834.

Nguyen, M. H. \& Yu, C. Y. (1998). In vitro comparative efficacy of voriconazole and itraconazole against fluconazole-susceptible and resistant Cryptococcus neoformans isolates. Antimicrob Agents Chemother 42, 471-472.

Odds, F. C. (1980). Laboratory evaluation of antifungal agents: a comparative study of five imidazole derivatives of clinical importance. J Antimicrob Chemother 6, 749-761.

Perfect, J. R., Marr, K. A., Walsh, T. J. \& 8 other authors (2003). Voriconazole treatment for less-common, emerging, or refractory fungal infections. Clin Infect Dis 36, 1122-1131.

Perkins, A., Gomes-Lopez, A., Mellado, E., Rodrigues-Tudela, J. L. \& Cuenca-Estrella, M. (2005). Rates of antifungal resistance among Spanish clinical isolates of Cryptococcus neoformans var. neoformans. J Antimicrob Chemother 56, 1144-1147.

Petraitiene, R., Petraitis, V., Groll, A. H. \& 7 other authors (2001). Antifungal activity and pharmacokinetics of posaconazole ( $\mathrm{SCH}$ 56592) in treatment and prevention of experimental invasive pulmonary aspergillosis: correlation with galactomannan antigenemia. Antimicrob Agents Chemother 45, 857-869.

Pfaller, M. A., Zhang, J., Messer, S. A., Brandt, M. E., Hajjeh, R. A., Jessup, C. J., Tumberland, M., Mbidde, E. K. \& Ghannoum, M. A. (1999). In vitro activities of voriconazole, fluconazole, and itraconazole against 566 clinical isolates of Cryptococcus neoformans 
from the United States and Africa. Antimicrob Agents Chemother 43, 169-171.

Pfaller, M. A., Messer, S. A., Houston, A., Mills, K., Bolmström, A. \& Jones, R. N. (2000). Evaluation of the Etest method for determining voriconazole susceptibilities of 312 clinical isolates of Candida species by using three different agar media. J Clin Microbiol 38, 3715-3717.

Pfaller, M. A., Walsh, T. J., Chaturvedi, V. \& 7 other authors (2001). Antifungal susceptibility testing: practical aspects and current challenges. Clin Microbiol Rev 14, 643-658.

Randhawa, H. S., Kowshik, T. \& Khan, Z. U. (2003). Decayed wood of Syzygium cumini and Ficus religiosa living trees in Delhi/ New Delhi metropolitan area as natural habitat of Cryptococcus neoformans. Med Mycol 41, 199-209.

Revankar, S. G., Kirkpatrick, W. R., McAtee, R. K., Dib, O. P., Fothergill, A. W., Redding, S. W., Rinaldi, M. G. \& Patterson, T. F. (1996). Detection and significance of fluconazole resistance in oropharyngeal candidiasis in human immunodeficiency virusinfected patients. J Infect Dis 174, 821-827.

Rex, J. H., Pfaller, M. A., Walsh, T. J. \& 8 other authors (2001). Antifungal susceptibility testing: practical aspects and current challenges. Clin Microbiol Rev 14, 643-658.

Saag, M. S., Graybill, R. J., Larsen, R. A., Papaas, P. G., Perfect, J. R., Powderly, W. G., Sobel, J. D. \& Dismukes, W. E. (2000). Practice guidelines for the management of cryptococcal diseases. Clin Infect Dis 30, 710-718.

Schwarz, P., Dromer, F., Lortholary, O. \& Dannaoui, E. (2006). Efficacy of amphotericin $B$ in combination with flucytosine against flucytosine-susceptible or flucytosine-resistant isolates of Cryptococcus neoformans during disseminated murine cryptococcosis. Antimicrob Agents Chemother 50, 113-120.

Vandenbossche, I., Vaneechoutte, M., Vandevenne, M., De Baere, T. \& Verschraegen, G. (2002). Susceptibility testing of fluconazole by the NCCLS broth macrodilution method, E-test, and disk diffusion for application in the routine laboratory. J Clin Microbiol 40, 918-921.

Van Duin, D., Cleare, W., Zaragoza, O., Casadevall, A. \& Nosanchuk, J. D. (2004). Effects of voriconazole on Cryptococcus neoformans. Antimicrob Agents Chemother 48, 2014-2020.

Warnock, D. W., Johnson, E. M. \& Rogers, T. R. (1998). Multicentre evaluation of the Etest method for antifungal drug susceptibility testing of Candida spp. and Cryptococcus neoformans. J Antimicrob Chemother 42, 321-331.

Yildiran, S. T., Fothergill, A. W., Sutton, D. A. \& Rinaldi, M. G. (2002). In vitro susceptibilities of cerebrospinal fluid isolates of Cryptococcus neoformans collected during a ten-year period against fluconazole, voriconazole and posaconazole (SCH56592). Mycoses 45, 378-383. 